\title{
Cumulative Risk Exposure and Social Isolation as Correlates of Carer and Child Mental Health During the COVID-19 Pandemic: An Online Study with Families from Various Europeans Countries
}

\author{
Ana Isabel Pereira ${ }^{1}\left[\right.$ D Peter Muris ${ }^{2} \cdot$ Magda Sofia Roberto $^{1} \cdot$ Paul Stallard $^{3} \cdot$ Luis-Joaquin Garcia-Lopez $^{4}$. \\ Bogdan Tudor Tulbure ${ }^{5} \cdot$ Ioana Podina $^{6} \cdot$ Ellin Simon $^{7} \cdot$ Marlene Sousa $^{8} \cdot$ Luísa Barros $^{1}$
}

Accepted: 15 August 2021 / Published online: 29 August 2021

(c) The Author(s), under exclusive licence to Springer Science+Business Media, LLC, part of Springer Nature 2021

\begin{abstract}
This study adopted a cumulative risk approach to examine the relations between various domains of risk factors (i.e., social isolation and home confinement, other pandemic-related risk factors, and pre-existing psychosocial risk factors) and carers' and children's mental health during the first wave of the COVID-19 pandemic in Europe. The sample consisted of 1475 carers of 6- to 16-year-old children and adolescents residing in five European countries (Portugal, United Kingdom, Romania, Spain, and The Netherlands) who completed an online survey. The results showed that each of the three domains of adversity accounted for unique variation in carers' and children's mental health outcomes. Also, the results indicated that pre-existing psychosocial risk factors moderated the relationship between pandemic-related risk factors and children and carers' anxiety and between social isolation and confinement and carers' well-being. Simple slopes analysis suggested a stronger relationship between these domains of adversities and mental health outcomes in already more vulnerable families. It is important to consider the implications of social isolation measures and confinement for families' mental health, paying special attention to families with pre-existing psychosocial vulnerabilities.
\end{abstract}

Keywords COVID-19 · Social isolation and home confinement · Pre-existing psychosocial risks · Mental health $\cdot$ Children, adolescents, and carers

\section{Introduction}

The coronavirus disease (COVID-19) outbreak began in 2019 and rapidly spread to Europe and other parts of the globe, leading the World Health Organization to eventually declare a global pandemic situation [1]. To combat the

Ana Isabel Pereira

aipereira@psicologia.ulisboa.pt

1 CICPSI, Faculdade de Psicologia, Universidade de Lisboa, Alameda da Universidade, 1649-013 Lisbon, Portugal

2 Maastricht University, Maastricht, The Netherlands

3 Department for Health, University of Bath, Bath, UK

4 University of Jaén, Jaén, Spain

5 West University of Timişoara, Timişoara, Romania

6 Bucharest University, Bucharest, Romania

7 Open University, Heerlen, The Netherlands

8 ProChild CoLAB, Guimarães, Portugal pandemic and control the spread of COVID-19, the World Health Organization and most governments recommended confinement measures that encouraged or even forced most of the population to "stay at home" for several weeks or months [2]. However, it has been noted that these public health measures, although effective in preventing the spread of the virus, have the potential to impair the mental health of the population [3]. The present study will explore the association between social isolation, other adversities related to the pandemic, and pre-existing psychosocial risk factors and carers' and children's mental health in five European countries.

This study adopts a developmental psychopathology framework [4]. The pandemic outbreak can be seen as a natural experiment [5] that enables us to study how significant changes in children's and carers' contexts interact with already existing psychosocial vulnerabilities to explain adaptive and maladaptive mental health outcomes. 


\section{Social Isolation During the COVID-19 Pandemic}

One of the major consequences of the pandemic and subsequent social distancing measures was the physical isolation from friends, extended family, and other significant figures. For children and adolescents, social distance measures may have had even more impact due to the developmental significance of peers, especially during adolescence $[6,7]$. Previous research has demonstrated a deleterious impact of loneliness on mental health. Although social isolation is not equivalent to loneliness [7], it can be expected that a reduction of social contacts will increase feelings of loneliness. The results of a systematic review of 63 studies [8] examining the impact of loneliness in previously healthy children showed clear cross-sectional and prospective associations between loneliness and mental health problems, notably the internalizing problems of anxiety and depression. Additionally, the duration of loneliness was a particularly strong predictor of mental health problems.

Nevertheless, social isolation during the COVID-19 pandemic may have distinctive characteristics and acquire a different meaning than the subjective feelings of loneliness or social isolation caused by other situations. During the pandemic, social isolation was instigated by the authorities, and as such, it was a common experience for all children in the community, experienced within the context of other significant threats [8]. Also, being physically isolated from friends, extended family, and other community members does not mean that one is fully socially isolated because there are other ways to maintain social contact. Moreover, during home confinement, distancing from friends and other relatives may also lead to more physical, and in some cases, emotional proximity with regular household members [9].

\section{Threatening Events and Acute Stress During the COVID-19 Pandemic}

Isolation was not the only potential adversity that families and children faced during the COVID-19 pandemic. Confinement measures led to a significant modification of families' lifestyles and routines [10]. Many carers had to work from home, and most children also stayed at home, with school-aged children participating in distance learning. These events potentially increased the burden and daily stress for carers who had to manage simultaneously work responsibilities, domestic duties, and supporting their children's learning activities. Also, several people faced the threat of losing their job or suffered a decrease in income and financial security. Finally, families perceived their life and health threatened, and some families suffered from the illness or even found themselves grieving for the death of significant ones. In some cases, children and parents were separated for some time due to the suspicion of illness or professional obligations (e.g., healthcare or frontline workers who were more exposed to the virus and chose to live separate from their families).

All these co-occurring adversities and demands may have created stress at multiple levels (i.e., intrapersonal, family, community) and challenged the most basic systems of adaptation, including emotional security, sensitive caregiving, self-efficacy, hope, family routines and organisation, and school and community involvement [11]. The exposure to multiple adversities associated with the COVID-19 pandemic is similar to that noted during other natural disasters. In these situations, families are confronted with a constellation of multiple risks rather than one isolated adverse event [12]. With this in mind, the present study adopted a cumulative risk approach [13] to examine the relations between various risk factors associated with the COVID-19 pandemic and carers' and children's mental health. In this study, we define risk factor as a correlate that has the potential to precede a negative outcome [14], but we need to keep in mind that given the cross-sectional design, we cannot infer any causality in the relations between pandemic-related risks and carers' and children's mental health.

\section{Pre-existing Psychosocial Risk Factors}

To understand the negative impact of the social disruption due to COVID-19, it is also necessary to consider pre-existing adverse family conditions and vulnerabilities [15], such as low family income, low educational levels, household overcrowding, chronic health conditions, and parents' mental health problems. The impact of such risk factors was demonstrated in Rutter et al.'s [16] study with children and families from the Isle of Wight. The results showed that an accumulation of contextual risks (e.g., low socioeconomic status, marital conflict, household overcrowding, maternal psychiatric disorder) led to an increased likelihood of negative outcomes. More specifically, children exposed to only one psychosocial risk did not necessarily show adverse outcomes, but those who faced a combination of multiple contextual risk factors had a significantly higher risk for developing mental health problems $[17,18]$.

\section{Current Study}

Previous studies conducted within the context of the COVID-19 pandemic have documented a negative impact on children's and parents' mental health, especially for anxiety problems, depression, and posttraumatic stress disorder $[4,10,19]$. Nevertheless, considerable variability in how 
families are affected by the COVID-19 can be expected [12]. Some families will be more vulnerable to the impact of the stress and hardship associated with the pandemic based on pre-existing vulnerabilities and cumulative risk factors $[15$, 20].

The main goal of the current study was to examine the relations among social isolation and home confinement, other adversities associated with the COVID-19 pandemic, and cumulative pre-existing psychosocial risk factors on the one hand, and carers' and children's mental health (as reported by carers) on the other hand. We also explored whether pre-existing psychosocial adversities moderate the effects of social isolation and other pandemic-related adversities on mental health outcomes. This work expands our knowledge in several ways. First, the specific associations of various domains of risk to children's and carers' mental health outcomes will be examined. Adopting a multiple risk factors approach [13], three domains of adversities will be considered: (1) social isolation and home confinement, (2) other pandemic-related adversities, like health threats or carers' overload, and (3) pre-existing psychosocial adversity. The inclusion of a large sample from various European countries will increase the diversity of exposure to pandemic-related risks, thereby facilitating the study of the associations between various risk factors and mental health outcomes. Also, to the best of our knowledge, this is the first study to examine the moderating role of pre-existing psychosocial risk factors in the relationship between the adversities associated with COVID-19 and children's and carers' mental health.

We hypothesised that social isolation and home confinement, cumulative pandemic-related risk factors, and cumulative pre-existing psychosocial risk factors account for unique variation in carers' and children's mental health-related outcomes. We also hypothesised that pre-existing psychosocial risk factors have a moderation effect on the relation between pandemic-related risk factors and mental health outcomes, which would indicate a greater negative impact of the pandemic in already more vulnerable families.

\section{Method}

\section{Participants}

The sample consisted of 1,475 carers of 6- to 16-year-old children and adolescents. Socio-demographic characteristics are presented in Table 1. The participants resided in one of the five European countries where the study was conducted: United Kingdom (33.5\%), Portugal (34.4\%), Romania (22.4\%), Spain (5.6\%), and the Netherlands (4.1\%). Most of the participants were parents $(87.1 \%$ mothers, $11.7 \%$ fathers, and $1.2 \%$ other carers), worked full-time (62.5\%), lived with
Table 1 Socio-demographic characteristics

\begin{tabular}{|c|c|c|}
\hline & \multicolumn{2}{|c|}{$\begin{array}{l}\text { Total sample } \\
(N=1475)\end{array}$} \\
\hline & $n$ & $\%$ \\
\hline \multicolumn{3}{|l|}{ Country } \\
\hline UK & 508 & 34.4 \\
\hline Portugal & 494 & 33.5 \\
\hline Romania & 330 & 22.4 \\
\hline Spain & 82 & 5.6 \\
\hline The Netherlands & 61 & 4.1 \\
\hline \multicolumn{3}{|l|}{ Carer } \\
\hline Mothers & 1285 & 87.1 \\
\hline Father & 173 & 11.7 \\
\hline Others & 17 & 1.2 \\
\hline \multicolumn{3}{|l|}{ Carer's age } \\
\hline$<30$ & 32 & 2.3 \\
\hline $30-39$ & 488 & 33.1 \\
\hline $40-49$ & 804 & 54.5 \\
\hline$>50$ & 149 & 10.1 \\
\hline \multicolumn{3}{|l|}{ Carer's professional situation } \\
\hline In full-time employment & 922 & 62.5 \\
\hline In Part-time employment & 303 & 20.5 \\
\hline Full-time mother/father & 110 & 7.5 \\
\hline Other situations & 140 & 9.5 \\
\hline Carer living with father or mother of child & 1203 & 81.6 \\
\hline \multicolumn{3}{|l|}{ Children in the household } \\
\hline 1 & 546 & 37.0 \\
\hline 2 & 713 & 48.3 \\
\hline 3 or more & 216 & 14.7 \\
\hline $\begin{array}{l}\text { Carer's or child's nationality different from the } \\
\text { residing country }\end{array}$ & 41 & 2.8 \\
\hline Child's sex-male & 744 & 50.5 \\
\hline \multicolumn{3}{|l|}{ Child's age } \\
\hline $6-9$ & 682 & 46.2 \\
\hline \multirow[t]{2}{*}{$10-16$} & 793 & 53.8 \\
\hline & $\begin{array}{l}M=10.13 \\
S D=2.92\end{array}$ & \\
\hline
\end{tabular}

the other parent of the child (81.6\%), and had one or two children (85.3\%). The sample had a balanced distribution regarding children's gender (50.5\% male, $49.5 \%$ female) and age (46.2\% 6- to 9-year-olds, $53.8 \% 10$ - to 16 -year-olds).

\section{Measures}

\section{Background Characteristics}

Carers completed several questions regarding socio-demographic characteristics (e.g., age, gender, nationality) and household composition (e.g., household composition, age of siblings). 


\section{Outcome Measures}

Child's Anxiety The Revised version of Screen for Child Anxiety Related Emotional Disorders (SCARED-R) [21] measures children's anxiety symptoms. In this study, the parent version was used, consisting of 69 items covering various symptoms domains (e.g., social anxiety, separation anxiety, specific phobias, generalised anxiety, panic disorder, posttraumatic stress). Carers are asked to rate their child's behaviour during the last month on a Likert scale ranging from 0 (never or almost never) to 2 (often). The total score that was used in the present study appeared to be highly reliable (Cronbach's $\alpha=0.95$ ).

Child's Well-Being The KIDSCREEN-10 [22] measures children's quality of life. Carers are asked to score 10 items, covering physical and psychological well-being, relationships with peers, and school functioning. Each item is rated on a five-point Likert scale $(1=$ not at all or never, $5=$ extremely or always) considering the child's behaviour during the last week. The total score displayed acceptable internal consistency $(\alpha=0.76)$.

Carer's Anxiety The 7-item Generalised Anxiety Disorder scale (GAD-7) [23] measures the frequency of anxiety symptoms in adults. Individuals rate each item on a four-point Likert scale $(1=$ not at all, $4=$ nearly every day $)$ considering the period of the last 2 weeks. Higher scores indicate higher levels of anxiety. In the current sample, the reliability coefficient of the GAD-7 was good $(\alpha=0.89)$.

Carer's Well-Being The Warwick-Edinburgh Mental WellBeing Scale (WEMWBS) [24] consists of 14 items measuring individuals' wellbeing. Based on the last 2 weeks, participants rated each item on a five-point Likert scale ( $1=$ none of the time, $5=$ all of the time). In the present study, the internal consistency of this measure was excellent $(\alpha=0.93)$.

\section{Dimensions of Risk Factors}

Index of Children's Social Isolation and Home Confinement Carers reported the degree of social isolation and home confinement resulting from the COVID-19 pandemic. This index consisted of four items describing the frequency of going out, moderate or vigorous exercise, distance or inperson contact with family members, and distance or in-person contact with friends in the previous week. Items were scored on a five-point Likert scale $(1=$ daily or almost daily; $2=4$ or 5 days a week; $3=2$ or 3 days a week; $4=1$ day a week; $5=$ never) and combined in such a way that higher scores indicated higher levels of social isolation and home confinement.
Index of Pre-existing and Pandemic-Related Individual Risk Factors Carers were asked several questions to assess potential psychosocial risk factors and risk factors related to the family situation during the pandemic. The first section of the questionnaire included questions regarding pre-existing psychosocial risks based on the cumulative risk literature $[17,18]$ : carers' level of education (various categories for each of the countries due to differences in the national educational and school systems), living arrangements (living with the father/mother of the child, living with a partner who is not the father/mother of the child, raising a child without a partner), if anyone in the household was diagnosed and/ or treated for behavioural, emotional, and/or developmental problems (yes/no), if anyone in the household had a chronic or severe medical condition (yes/no), how many children (aged 0-17 years) were living in the household, how many adults (older than 18 years) were living in the household, and the number of rooms in the home/flat (not including bathroom, toilet, and kitchen). Another section of the questionnaire addressed risk factors related to the family situation during the pandemic. This section included the following questions: interruption of contact with carers (yes/no), reduction of income (yes/no), if the child had COVID-19 (no, had COVID-19 or suspected to have), if someone that the child knew well was infected with COVID-19 (no, had COVID-19 or suspected to have), if there were members of the household that were COVID-19 key workers (e.g., doctors, nurses, police; yes/no), how many adults in the household worked exclusively from home, age of the child's siblings (0-2 years, $3-5$ years, $6-12$ years, $13-17$ years). A summary of individual risk factor variables and their relationship with children's and carers' anxiety and well-being scores is provided in the supplementary materials, Table S1.

\section{Data Collection Procedure}

This cross-sectional online study was part of a larger research project on the mental health of carers and children during the first COVID-19 outbreak. The ethical committees of the participating institutions reviewed and approved the study protocol. There was no conflict of interest for any of the researchers involved in this study.

The online survey was developed in the Qualtrics platform and hosted by the Faculty of Psychology, University of Lisbon. The participants' recruitment in all countries was conducted through various channels: newspapers, social media, email, and institutional advertising. Carers of 6- to 16-year-old children were invited to participate in the study. After the carers had provided informed consent, they were instructed to complete the online survey. When participants had more than one child in this age range, they were asked to focus on one of the children. 
Data was collected immediately after the first wave of the highest incidence of new cases and mortality rates in each country, between May 1 and July 7, 2020, with 75\% of participants completing the questionnaire online during May. During this period, all five countries faced a total or partial lockdown, involving multiple mandatory measures, including enforcement of social distancing, school closures, remote working for parents, and home confinement.

\section{Data Analyses}

We performed hierarchical multiple linear regression analysis to evaluate the relationships between the study outcomes (carers' and children's well-being and anxiety scores) and various covariates (i.e., social isolation and home confinement, pandemic-related risks, and pre-existing psychosocial risks) as well as their moderating effects. First, in Step 1, we entered the child's socio-demographic variables (age and gender). Then, we entered the three dimensions of risk factors as covariates, followed, in Step 3, by the three interaction terms (i.e., cumulative psychosocial risk $\times$ cumulative pandemic related risk, cumulative psychosocial risk $\times$ social isolation and home confinement, and cumulative pandemicrelated risk $\times$ social isolation and home confinement). Following the recommendations of Cohen, Cohen, West, and Aiken [25], (1) covariates were centered at their means before entering them into the regression analyses to eliminate non-essential multicollinearity and ease coefficient interpretations; (2) for models with significant interaction terms, effects were plotted using Johnson-Neyman intervals to explore whether the regression slopes of the study outcomes on a given covariate changed at specific values of the moderator and became significant $(p<0.05)$ [26].

Assumptions underlying multiple linear regression models were checked. Specifically, we evaluated the existence of homoscedasticity (spread-location plot) as well as residuals independence (Durbin-Watson test statistic should be close to 2 to suggest an autocorrelation of 0 ) and normality (normal $q-q$ plot). For outliers screening, we visually inspected the residuals versus leverage plot to evaluate the presence of extreme values (leverage) with a potentially influential role on the regression estimates (values surpassing Cook's distance barriers). Finally, variance inflation values (VIF) less than 5 suggested there was no problem with multicollinearity [27]. Descriptive analyses were performed using SPSS (version 26, SPSS Inc., Chicago, IL). Linear regression models were estimated using R [28], with assumptions being checked with the package ggfortify [29, 30]. Simple slope analysis and interaction plotting were performed with jtools [31] and interactions [32] packages.

\section{Results}

\section{Construction of the Two Indexes of Cumulative Risk Factors: Psychosocial Pre-existing Risk Factors and Pandemic-Related Risk Factors}

The top panel of Table 2 presents the criteria for each risk factor selected for the cumulative psychosocial risk index. This index was constructed following two steps. First, we

Table 2 Cumulative risk individual indicators, defining criteria and percentage of participants meeting the criteria

\begin{tabular}{|c|c|c|}
\hline Indicator & Description of criteria & $\%$ \\
\hline \multicolumn{3}{|l|}{ Previous psychosocial risk } \\
\hline Carer education & No university degree & 20.8 \\
\hline Single parent & Living without a partner & 12.7 \\
\hline Mental health problems & $\begin{array}{l}\text { At least one member of the household been diagnosed } \\
\text { and/or treated for behavioral, emotional or } \\
\text { developmental problems }\end{array}$ & 18.3 \\
\hline Chronic health problems & $\begin{array}{l}\text { At least one member of the household with a chronic or } \\
\text { severe health medical condition }\end{array}$ & 19.5 \\
\hline Overcrowding & Two or more persons per room & 4.1 \\
\hline \multicolumn{3}{|c|}{$\begin{array}{l}\text { Cumulative psychosocial risk } \\
0 \text { risks }=47.3 \%, 1 \text { risk }=34.7 \%, 2 \text { risks }=14 \%, 3 \text { risks }=3.6 \%, 4 \text { risks }=0.5 \%\end{array}$} \\
\hline \multicolumn{3}{|l|}{ Pandemic related risk } \\
\hline Child infected with COVID-19 & Child had COVID or suspected to have & 5.2 \\
\hline Someone close infected with COVID-19 & Someone close had COVID or suspected to have & 14.6 \\
\hline Only one adult working exclusively from home & $\begin{array}{l}\text { Only one adult in the household working exclusively } \\
\text { from home }\end{array}$ & 42.6 \\
\hline Having siblings in school age (6-12 years) & $\begin{array}{l}\text { Having at least a sibling in the age range from } 6 \text { to } \\
12 \text { years }\end{array}$ & 31.4 \\
\hline $\begin{array}{l}\text { Cumulative pandemic related risk } \\
0 \text { risks }=34.0 \%, 1 \text { risk }=45.7 \%, 2 \text { risks }=17.0 \%, 3\end{array}$ & & \\
\hline
\end{tabular}


coded each risk factor dichotomously to indicate the presence (1) or absence of risk (0). For example, overcrowding, a continuous risk factor (the number of household members per room in the house), was dichotomised, using a cut-off of two or more persons per room [18]. Second, we compared the mean scores on carers' and children's mental health outcome measures between risk and non-risk groups to decide which factors to include in the psychosocial risk factor index. If there was a significant difference between the groups, that individual risk factor was included in the cumulative index. The precise results of these analyses are shown in the supplementary materials, Table S1. The preexisting psychosocial risk factors index eventually included the following five risk factors: lower education of the carer, raising a child without a partner, mental health problems in household members, chronic health conditions in household members, and living in an overcrowded house.

A similar procedure was used to construe the cumulative pandemic-related risk factors index. These variables were already scored on a dichotomous scale (absence of risk $=0$, presence of risk $=1$; see lower panel of Table 2). The following four risk factors were included in the cumulative pandemic-related risk factors index: two risk factors related to health threat (the child was ill or suspected to be ill with COVID-19; someone close was ill or suspected to be ill with COVID-19), and two risk factors related to overload of the carer (school-aged siblings; one carer working exclusively from home).

\section{Preliminary Analyses}

Almost all families were in home confinement. Specifically, 99\% of the children were no longer allowed to go to school: $47.2 \%$ had to stay at home for more than 9 weeks, $51.0 \%$ between 5 and 8 weeks, and $1.8 \%$ for 4 weeks or less. A significant part of the adults in the household was working exclusively from home ( $42.6 \%$ one adult, $22 \%$ two or more adults). Concerning family income, $54.7 \%$ of participants reported no income reduction. However, $29.2 \%$ of the families reported an income reduction of up to $30 \%$, while $16.1 \%$ had an income reduction of even more than $30 \%$.

Table 3 presents means, standard deviations, and correlations among the risk dimension variables and carers' and children's outcomes. In general, there was a substantial percentage of carers in the current sample who showed negative mental health outcomes. Using normative cut-off values for GAD-7 [33] and the WEMWBS [34], 28.7\% of the carers in this sample presented anxiety levels in the clinical range while $19.5 \%$ of them displayed scores that were indicative of a low well-being. Carers also reported that the quality of life of their children was considerably lower than normative data as obtained in an international survey conducted in 11 countries $(M=41.37, S D=8.71, n=1.475$ versus $M=49.74$, $S D=10.14, n=8.072 ; t(9545)=29.76, p<0.001$, Cohen's $d=0.89$ ) [22]. Concerning children's anxiety level, the absence of international normative data and cut-off scores for the parents' version of the SCARED-R prevented us from analysing the current data in this respect.

Correlations between risk factors and mental health outcomes were all statistically significant, in the low to moderate range, but in the expected direction: higher risk was associated with higher anxiety ( $r$ 's between 0.08 and 0.27 ) and lower well-being ( $r$ 's between -0.12 and -0.26 ) in both children and carers. Correlations among carers' and children's mental health outcomes were also as hypothesized and fell in the moderate to high range (with negative $r$ 's between -0.29 to -0.54 and positive $r$ 's of 0.41 and 0.49). Concerning the correlations among risk dimensions, it should be noted that only the correlation between social isolation and home confinement and pre-existing psychosocial risks was significant $(r=0.10)$.

\section{Regression Analyses Explaining Well-Being from Risk Factors}

Results for the regression analyses with well-being outcomes as the dependent variable are shown in Table 4. In the regression analysis predicting children's well-being scores,

Table 3 Descriptive statistics and correlations among the main study variables $(n=1475)$

\begin{tabular}{|c|c|c|c|c|c|c|c|c|}
\hline & $M$ & $S D$ & 2 & 3 & 4 & 5 & 6 & 7 \\
\hline 1. Social isolation & 2.48 & 0.80 & -0.05 & $0.10 * * *$ & $0.14 * * *$ & $-0.19 * * *$ & $0.15^{* * *}$ & $-0.26^{* * *}$ \\
\hline 2. Pandemic-related risk & 0.94 & 0.81 & & 0.04 & $0.08 * *$ & $-0.12 * * *$ & $0.09 * *$ & $-0.16 * * *$ \\
\hline 3. Psychosocial risk & 0.75 & 0.86 & & & $0.17 * * *$ & $-0.18 * * *$ & $0.27 * * *$ & $-0.16 * * *$ \\
\hline 4. GAD-7 Carer anxiety & 6.01 & 4.76 & & & & $-0.54 * * *$ & $0.41 * * *$ & $-0.32 * * *$ \\
\hline 5. WEMWBS Carer well-being & 48.70 & 9.40 & & & & & $-0.29 * * *$ & $0.49 * * *$ \\
\hline 6. SCARED-R Child anxiety & 29.73 & 19.73 & & & & & & $-0.39 * * *$ \\
\hline 7. KIDSCREEN-10 Child well-being & 35.13 & 5.21 & & & & & & \\
\hline
\end{tabular}

GAD-7 Generalised Anxiety Disorder 7-Item Scale, WEMWBS The Warwick-Edinburgh Mental Well-being Scale, SCARED-R Screen for Child Anxiety Related Emotional Disorders-Revised Version, KIDSCREEN-10 Health Questionnaire for Children and Young People-10 index

$* * * p<0.001 ; * * p<0.01 ; * p<0.05$ 
Table 4 Main results of the regression analyses in which children's and carers' well-being were outcomes and social isolation, pandemic-related, and psychosocial risk factors were covariates

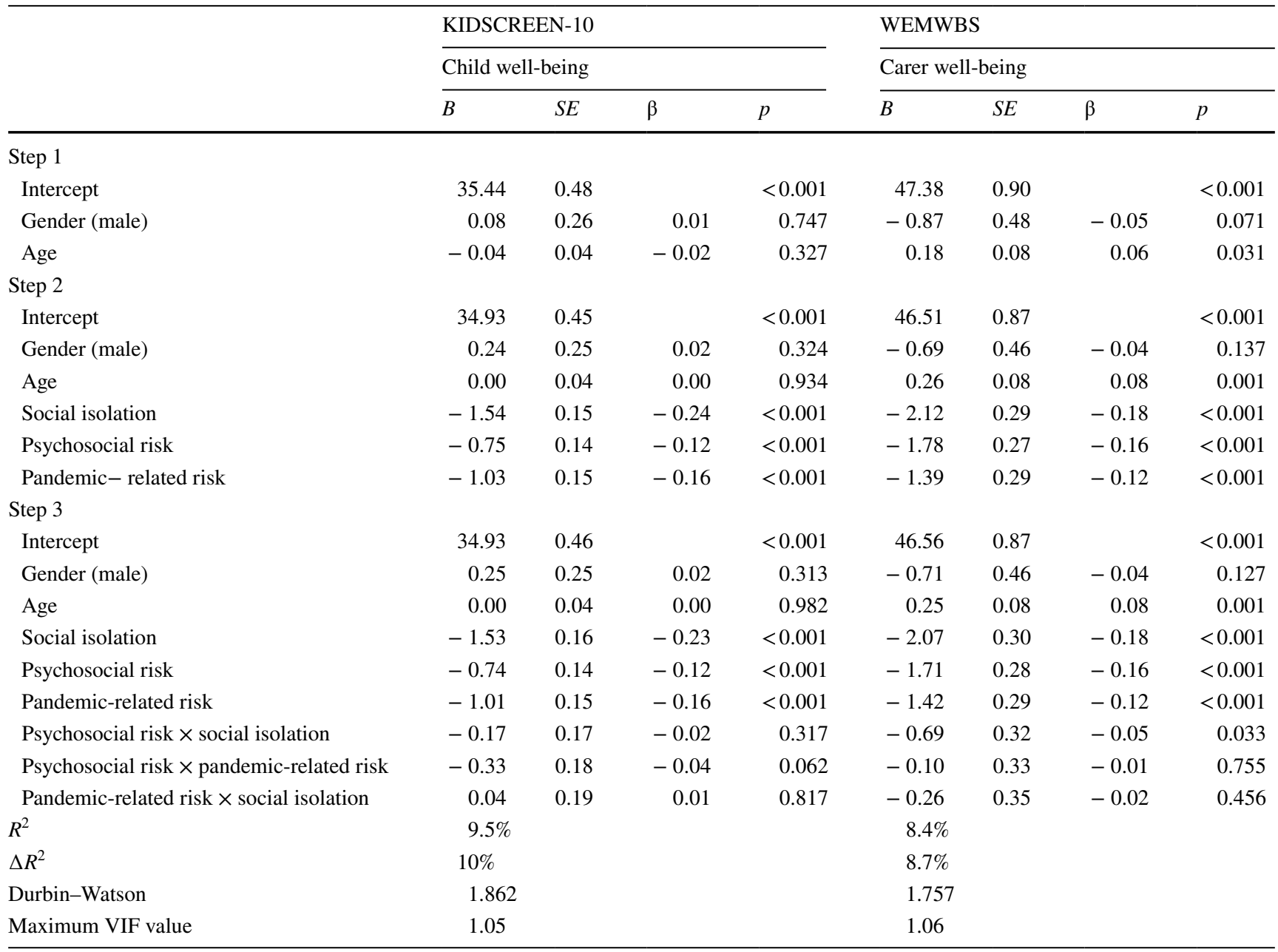

socio-demographic covariates did not account for a significant proportion of the variance at Step $1[F(2,1647)=0.52$, $p=0.595]$. At Step 2, the introduction of risk covariates explained $9.8 \%$ of the outcome variance $[F(5,1644)=35.84$, $p<0.001]$, contributing significantly and negatively to children's well-being scores. Finally, the introduction of the interaction terms in Step 3 explained an additional 0.2\% $[F(8,1641)=22.94, p<0.001]$ : between Steps 2 and 3 the $F$ change was non-significant $(p=0.244)$, with none of the interaction terms showing $p$-values lower than 0.05 .

In the regression analysis explaining carers' well-being scores, the age of the child significantly and positively explained well-being scores $[F(2,1495)=3.820, p=0.022$; $R^{2}=1 \%$ ]. Explained variance changed $8 \%$ at Step 2 with the inclusion of various risk factors $[F(5,1492)=27.25$, $p<0.001]$. As previously identified, a similar pattern was found with all three risks negatively and significantly explaining well-being scores. Finally, at Step 3 an additional $0.3 \%$ of variance was explained $[F(8,1489)=17.72$, $p<0.001]$. Despite the $F$ change not being significant between Steps 2 and $3(p=0.154)$, the interaction term between psychosocial risk and social isolation was significant $(p=0.033)$. This effect was plotted and is displayed in Fig. 1. Simple slopes analysis using Johnson-Neyman intervals $[-5.03,-1.21]$ revealed that social isolation had an effect on the carers' well-being when the psychosocial risk was higher than 0 . Thus, the negative slope of social isolation on well-being was significant for all levels of psychosocial risk and became steeper with increasing levels of psychosocial risk.

\section{Regression Analyses Explaining Anxiety from Risk Factors}

Table 5 shows the results of the regression models in which children's and carers' anxiety outcomes were explained from various risk factors and their interaction terms. In the regression analysis predicting children's anxiety scores, gender made a significant contribution at Step $1[F(2,1550)=4.98, p=0.007]$, explaining $1 \%$ of the 
Fig. 1 Simple slopes analysis representing the interaction of social isolation and pre-existing psychosocial risk in explaining carers' well-being

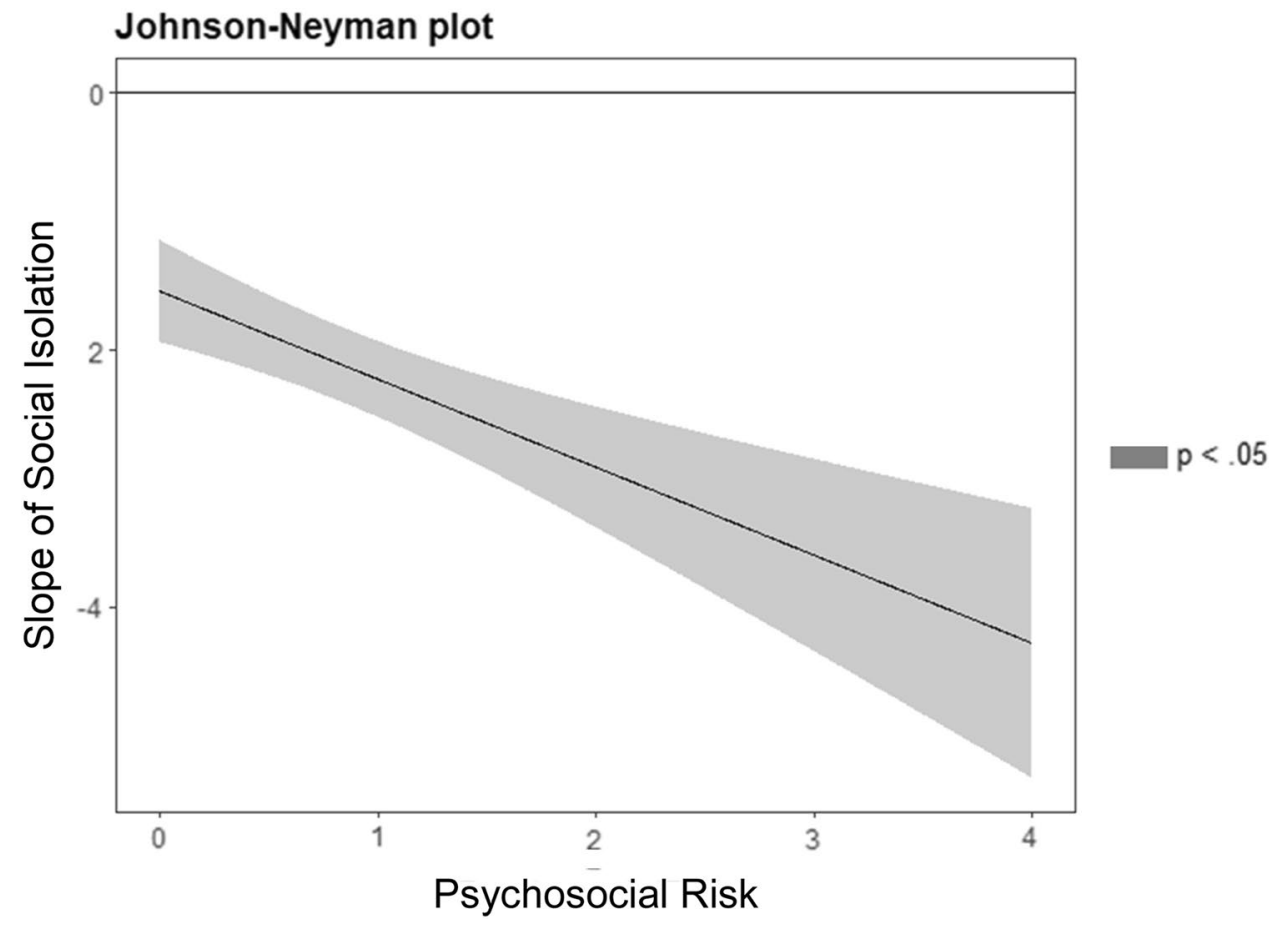

outcome variance. In Step 2, the risk factors explained an additional $9.2 \%$ of the variance $[F(5,1547)=33.58$, $p<0.001]$ with each of them making a significant and positive contribution to children's anxiety. The interaction term between psychosocial risk and pandemic-related risk was significant $(p<0.001)$ and explained an extra $1.1 \%$ of the variance at step $3[F(8,1544)=23.54, p<0.001]$. A simple slope analysis was performed, and the plot of the significant interaction is displayed in Fig. 2. The JohnsonNeyman interval for the analysis ranged between -0.27 and 0.28 , revealing a positive slope of the pandemicrelated risk on children's anxiety when the psychosocial risk was higher than 0.28 , with the regression line becoming steeper for higher levels of this risk.

In the regression analysis explaining carers' anxiety, the socio-demographic covariates did not explain a significant proportion of the variance at step $1[F(2,1510)=2.74$, $p=0.065]$, but the risk factors that were added at Step 2 explained an extra $5 \%$ of the variance $[F(5,1507)=17.39$, $p<0.001]$ : all three risk factors were positively and significantly associated with carers' anxiety. In step 3 , the interaction term between psychosocial risk and pandemic-related risk was also significant $(p=0.037)$, explaining an additional $0.3 \%$ of the variance $[F(8,1504)=11.53, p<0.001]$. Figure 3 displays the plot for this significant interaction. JohnsonNeyman interval $[-2.17,-0.03]$ revealed that the positive slope of pandemic-related risk on carers' anxiety was present for all psychosocial risk levels, with the regression line becoming steeper for higher levels of risk.

\section{Discussion}

The COVID-19 pandemic had a significant impact on the lives of families and communities across the world, creating significant challenges for the mental health of both carers and their children. The degree of exposure to pandemicrelated adversities and the individual and family response to these challenges may contribute to substantial variability in people's mental health during this period [10, 15]. The current study examined the associations between children's and carers' mental health during the first months of the COVID19 outbreak in five European countries and three domains of adversities and risk, namely social isolation and home confinement, other pandemic-related risks, and pre-existing psychosocial risks. In line with the premises of the developmental psychopathology framework [4, 14], this study also examined the moderating role of pre-existing psychosocial vulnerabilities in the relation between pandemic-related adversities and carers' and children's mental health outcomes [15].

As hypothesised, home confinement and isolation were associated significantly with children's anxiety and wellbeing. This result is consistent with earlier studies showing that children who have been quarantined are more likely to manifest higher levels of mental health problems [35]. Although there are several ways to maintain remote contact with friends and close family members such as grandparents (e.g., via skype, zoom, or facetime), these communication 
Table 5 Main results of the regression analyses in which children's and carers' anxiety were outcomes and social isolation, pandemic-related, and psychosocial risk factors were covariates

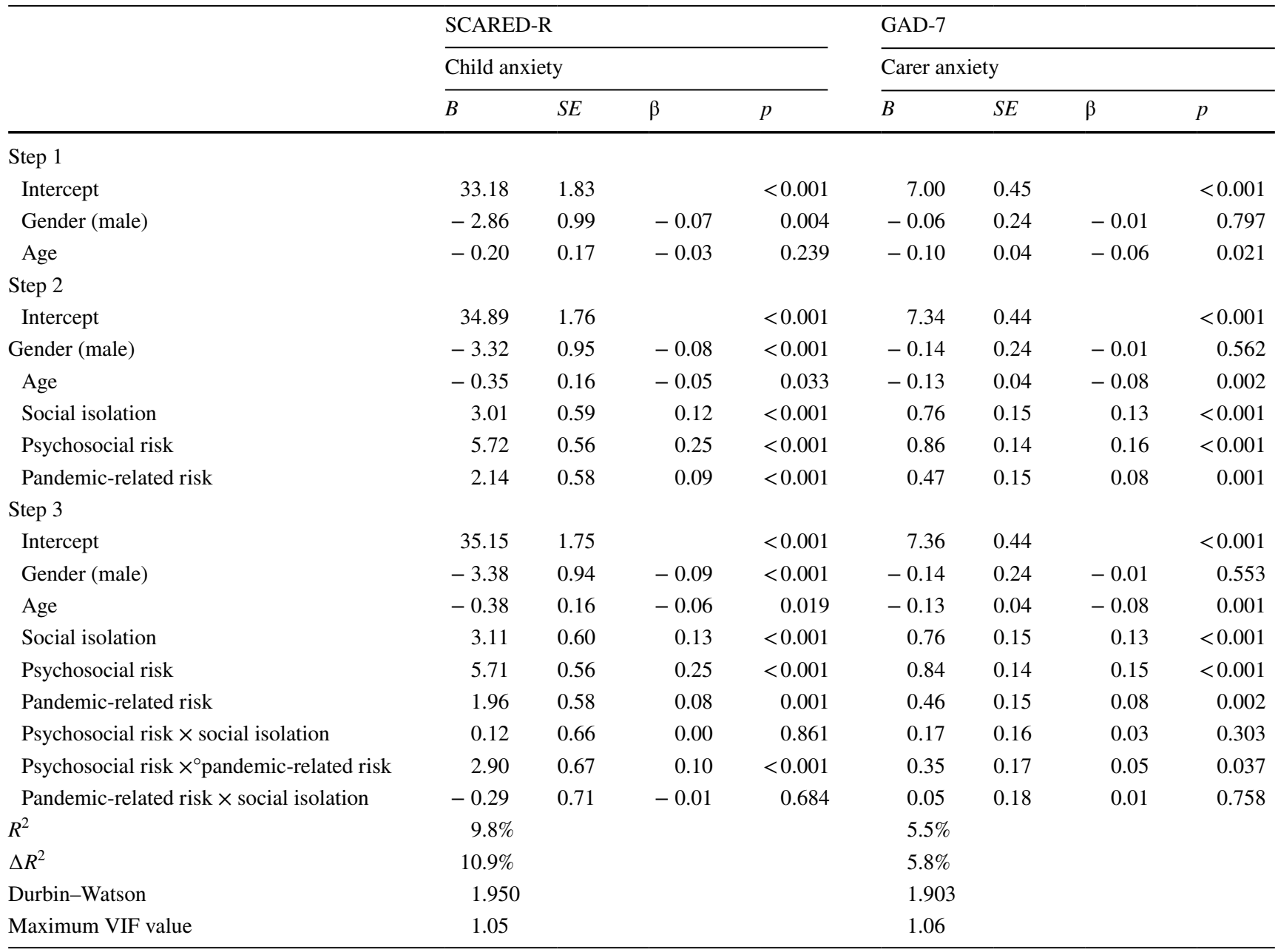

methods can be difficult for some children (e.g., shy children, those lacking appropriate technological equipment). In the current sample, $27.2 \%$ of the carers reported that their children had reduced contact (only once a week or none) with friends and $30.2 \%$ reported children's reduced contact with the extended family. Further, it is important to note that many children and adolescents were deprived of in-person contact with friends, even when maintaining some remote contact. Social interactions among children often occur while being involved in physical play activities, which are impossible during distance communication. Especially for adolescents, the impossibility of physical contact with close friends and romantic partners might be perceived as a significant loss.

Besides isolation from extended family and friends, home confinement often implied other restrictions, namely not going outside and reduced physical activity. Recent studies on the impact of COVID-19 showed that the pandemic was associated with low physical activity levels, less overall outside time, and a high frequency of sedentary behaviours
$[36,37]$. In our sample, $23.3 \%$ of the carers reported that their child engaged in moderate or vigorous physical exercise only once a week or even less, while $26.9 \%$ reported that their child hardly participated in any outdoor activities. These results are worrisome, in particular when considering that home quarantine lasted for multiple weeks. The lack of physical exercise and playing outside can have negative consequences, not only for physical development and health but also for children's and adolescents' mental health [38].

Pre-existing psychosocial adversities were also associated with children's and adolescents' anxiety symptoms and wellbeing. The significant impact of social disadvantages on children's mental health is robust in the literature [39]. This effect was also evident in our sample, although families in extreme poverty or social fragility were not included. Social disadvantage can impact children's mental health directly, or indirectly through parents' emotional strain and disrupted family processes, including couple hostility, withdrawal, and harsh, inconsistent or uninvolved parenting [40]. 


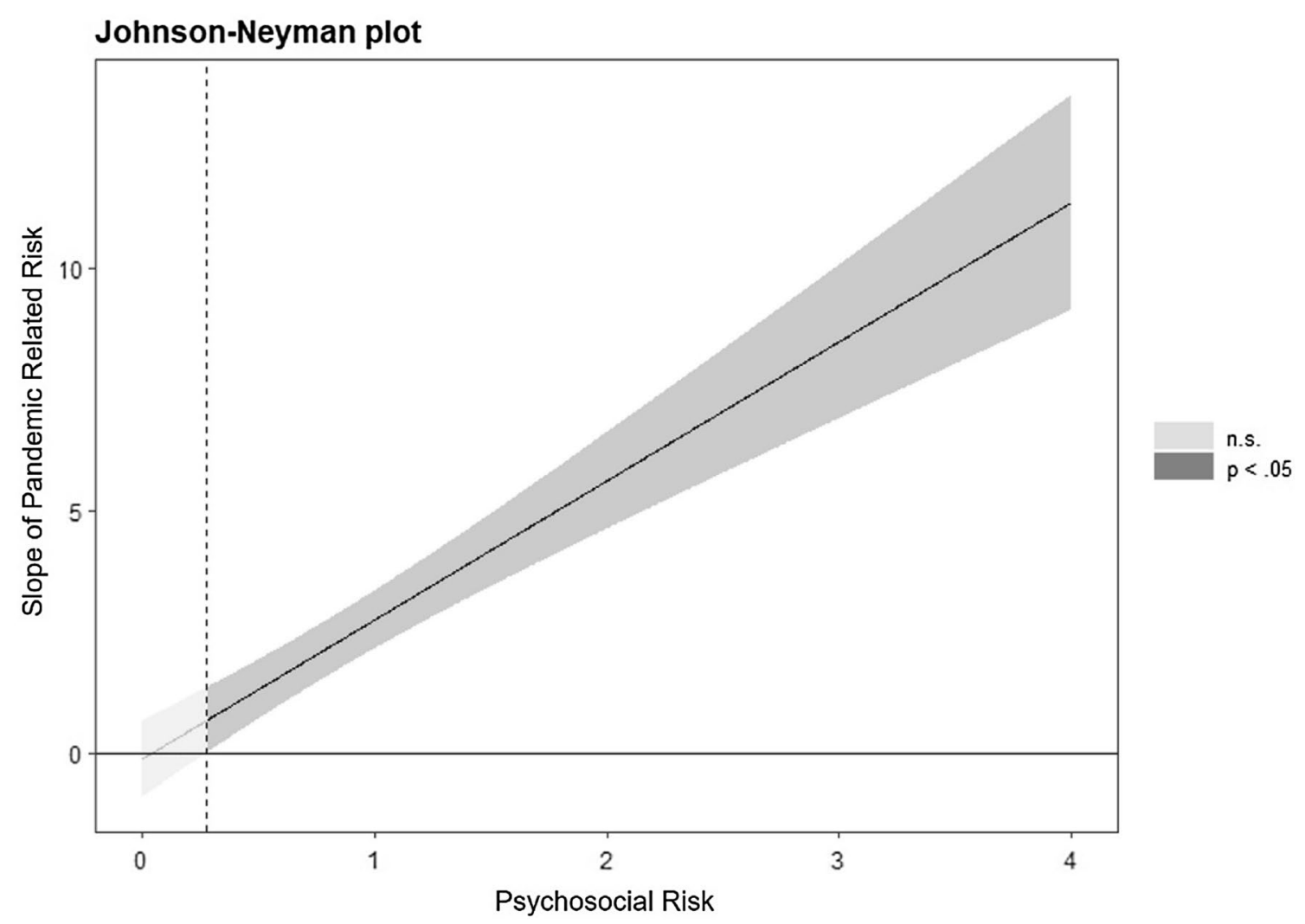

Fig. 2 Simple slopes analysis representing the interaction of pandemic-related and psychosocial risk factors in explaining children's anxiety

Finally, cumulative pandemic-related risks contributed significantly to children's and adolescents' mental health outcomes. This factor included various adversities associated with the COVID-19 pandemic, including health threats (someone in the family or close to the family was infected or suspected to be infected) and excessive burden for one of the carers (one parent/carer working from home and having to support and supervise one or more children in home schooling). The relation between pandemic-related adversities and children's anxiety was stronger for families with a higher level of pre-existing psychosocial risk factors. More precisely, youngsters belonging to families who were already exposed to psychosocial adversities were more vulnerable and likely to display increased anxiety levels when facing the stress resulting from the pandemic. In these circumstances, carers of more vulnerable families might have been more prone to display negative parenting and/or might have been less able to provide support to their children and teach them adequate coping strategies in order to buffer the negative impact of pandemic-related stressors.

Our results also showed that various risk domains independently contributed to carers' anxiety and well-being across all age groups. Furthermore, pre-existing cumulative psychosocial risks moderated the relationship between pandemic-related adversities and carers' anxiety. Again, the effects of pandemic-related adversities on parents' anxiety were more pronounced in vulnerable families which were already exposed to psychosocial risks (e.g., less financial resources, presence of household members with health conditions).

In general, home confinement appeared to have a significant negative impact on children's and carers' mental health. However, the significant interaction effect between social isolation and psychosocial risks on carers' well-being indicated that this situation was a particular burden for carers who already encountered difficulties in their life. Thus, preexisting psychosocial disadvantages, such as lower education, overcrowded houses, and higher demand for supporting other family members with mental or physical health problems, increase the negative impact of social isolation and home confinement. It seems plausible to assume that due to persistent pre-existing stressors families were already under a lot of stress and that the home confinement magnified this problem, resulting in a decrease of carers' well-being. This is especially problematic as carers' emotional distress can "spill over" into the relations with children through negative parenting practices [41].

Meanwhile, we should highlight that various risk factors only explained a relatively small percentage of the variance in carers' and children's mental health outcomes (i.e., 
Fig. 3 Simple slopes analysis representing the interaction of pandemic-related and psychosocial risk factors in explaining carers' anxiety

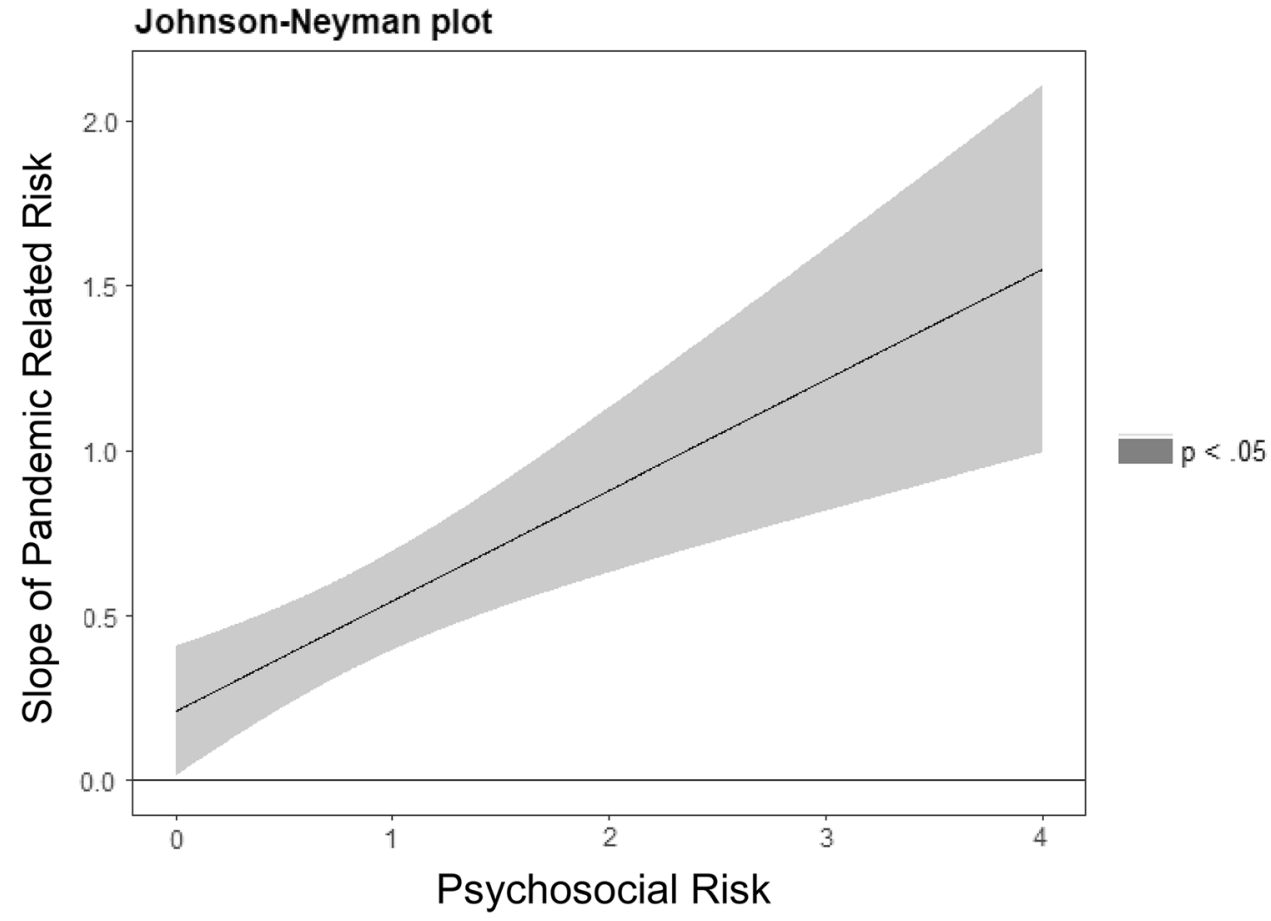

between 5 and 10\%). However, it should be borne in mind that our sample was not representative of the more vulnerable segments of the population (e.g., families in poverty, families with high violence) since this was an online study with a self-selected sample. In more vulnerable families, pandemic-related and psychosocial risks can be expected to have a more substantial impact, leading to a more severe disruption of carers' and children's functioning. Also, the cumulative risk indexes did not include proximal processes that could significantly impact children's mental health, like nurturance and parenting. Previous literature suggests that the impact of cumulative psychosocial risks on children's mental health may be primarily accounted for by these proximal factors [42].

The current study suffers from several limitations. First, the cross-sectional nature of the data prevents conclusions about the directions of effects in the associations found. Also, we only examined factors associated with children's and carers' short-term adjustment. Longitudinal studies are needed to explore the medium- and long-term impact of these risk factors and clarify the processes underlying them [43]. Second, we only collected carers' reports. This is particularly problematic for assessing children's and adolescents' internalizing problems, which are generally underreported by parents [44]. Third, this was a sample of highly educated carers, limiting the generalisation of the results to less educated and more disadvantaged families. Finally, although the sample size was large, it included participants from five different countries. The decision to perform ordinary least squares analyses has the disadvantage of not allowing to model data at the country level. The small number of unbalanced level-2 groups compromised the ability to perform multilevel modelling. Thus, the results should be interpreted with some caution given that that standard errors (not regression coefficients) may be biased [45, 46].

Notwithstanding these limitations, this study contributes to a further understanding of carers' and children's adaptation to the COVID-19 pandemic. Although home confinement is an effective measure to prevent the spread of the disease, it is crucial to consider its negative impact on children's and carers' mental health, especially if new restrictive measures are implemented. Children's home confinement and social isolation were associated with carers' and children's mental health outcomes. Children's social connections and physical activity are essential sources of development, affiliation, self-esteem, and social reward. When future developments again require similar public health measures, this mental health impact should be considered in public health authorities' decision-making.

On the other hand, if restrictive social measures are unavoidable, carers should be guided and supported to buffer the adverse effects of social isolation and maintain children's social interactions, using remote communication resources if necessary. Introverted children or children with fewer interpersonal resources may need more encouragement in this process. Schools and recreational structures can promote distance activities that can help children and youngsters to engage in social activities, enabling youth to interact with peers in small groups while conducting structured (e.g., working on group assignments) and unstructured activities 
(e.g., support groups). Considering the possibility of children from more vulnerable or at-risk families attending school at least for some hours each day during lockdown may be an alternative strategy to prevent more severe consequences. In addition, online schooling and social messages should encourage carers to keep children physically active, and governments should seriously consider encouraging some outdoor activities. These efforts could include disseminating information on home-based exercises for all ages through various channels (e.g., national TV, online resources).

Finally, these findings regarding the effects of cumulative risks reinforce the need to pay special attention, in the near future, to families for whom the combination of health and social adversities might have increased the impact of the pandemic. This attention is crucial during periods of restriction and afterwards, when children return to school and their regular daily activities. Assuring remote support for these children and families (e.g., school mentoring, telepsychology services) or special exemptions during the lockdown (e.g., allowing specific groups to continue in-person schooling, having some in-person mental health consultations). In addition, the implementation of mental health screening and prevention measures after the pandemic, may be crucial to guarantee their protection. The COVID-19 pandemic represents a global crisis for physical health, economic and social life, impacting children's and families' mental health in a way that will go beyond the phases of the active outbreak. It is essential to support children and families in building resources to face these adversities and pursue the research on how risk and protection processes may interact to construct resilience.

\section{Summary}

Families faced significant adversity as a result of the COVID-19 pandemic. This study adopted a multiple risk factors approach to examine the relations between carers' and children's mental health during the first wave of the COVID-19 pandemic in Europe. Various domains of risk, including social isolation and home confinement, other pandemic-related risk factors, and pre-existing psychosocial risk factors were examined in relation to carers' and children's anxiety and well-being. Considering that pre-existing vulnerabilities might play a role in the way individuals and families responded to the adversities associated with COVID-19, we also explored the moderating role of pre-existing psychosocial risk factors in the relationship between social isolation and other pandemic-related adversities and children's and carers' mental health.

Carers of 6- to 16-year-old children and adolescents residing in five European Countries (Portugal, United Kingdom, Romania, Spain, and The Netherlands) completed an online survey just after the first wave of the COVID-19 outbreak. The results showed that various risk domains independently contributed to carers' and children's mental health outcomes. Also, the negative impact of adversities associated with COVID-19 were more substantial for families with pre-existing psychosocial risk for children and parent's mental health outcomes. Therefore, it is crucial to consider the negative effects of public health measures such as home confinement and social isolation for children's and families' mental health, paying particular attention to those families with pre-existing psychosocial vulnerabilities and helping them build resources to recover from this global crisis.

Supplementary Information The online version contains supplementary material available at https://doi.org/10.1007/s10578-021-01233-3.

Funding We have no funding to declare.

Data Availability The dataset analysed in the current study is available from the corresponding author.

\section{Declarations}

Conflict of interest The authors have declared that they have no competing or potential conflicts of interest.

Ethical Approval All procedures were performed in studies involving human participants were in accordance with the ethical standards of the institutional committee and with the 1964 Helsinki declaration and its later amendments or comparable ethical standards.

\section{References}

1. WHO (2020) Director General's opening remarks at the media briefing on COVID-19, 11 March 2020. https://www.who.int/dg/ speeches/detail/who-director-general-s-opening-remarks-at-themedia-briefing-on-covid-19--11-march-2020

2. Matias T, Dominski FH, Marks DF (2020) Human needs in COVID-19 isolation. J Health Psychol 25(7):871-882

3. Chtourou H, Trabelsi K, H'Mida C, Boukhris O, Glenn JM, Brach M et al (2020) Staying physically active during the quarantine and self-isolation period for controlling and mitigating the covid-19 pandemic: a systematic overview of the literature. Front Psychol 11:1708

4. Cummings EM, Davies PT, Campbell SB (2000) Developmental psychopathology and family process: theory, research, and clinical implications. Guilford Press, New York

5. Bronfenbrenner U, Morris PA (2006) The bioecological model of human development. In: Lerner RM, Damon W (eds) Handbook of child psychology: theoretical models of human development. Wiley, New York, pp 793-828

6. Bukowski WM, Buhrmester D, Underwood M (2011) Peer relationships as a developmental context. In: Underwood M, Rosen L (eds) Social development. Guilford Press, New York, pp 153-179

7. Maria M (2020) No child is an island: sociability in times of social distancing. Eur Child Adolesc Psychiatry 29:901-902

8. Loades ME, Chatburn E, Higson-Sweeney N, Reynolds S, Shafran R, Brigden A et al (2020) Rapid systematic review: the impact of 
social isolation and loneliness on the mental health of children and adolescents in the context of COVID-19. J Am Acad Child Adolesc. https://doi.org/10.1016/j.jaac.2020.05.009

9. Stallard P, Pereira AI, Barros L (2021) Post-traumatic growth during the COVID-19 pandemic in carers of children in Portugal and the UK: cross-sectional online survey. BJPsych Open 7(1):e37

10. Miranda MD, da Silva Athanasio B, Sena Oliveira AC, Simoes-ESilva AC (2020) How is COVID-19 pandemic impacting mental health of children and adolescents? Int J Disaster Risk Reduct 51:101845

11. Masten AS, Barnes AJ (2018) Resilience in children: developmental perspectives. Children (Basel) 5(7):98

12. Clemens V, Deschamps P, Fegert JM, Anagnostopoulos D, Bailey S, Doyle M, Eliez S et al (2020) Potential effects of "social" distancing measures and school lockdown on child and adolescent mental health. Eur Child Adolesc Psychiatry 29:739-742

13. Evans GW, Li D, Whipple SS (2013) Cumulative risk and child development. Psychol Bull 139(6):1342-1396

14. Kraemer HC, Stice E, Kazdin A, Offord D, Kupfer D (2001) How do risk factors work together? Mediators, moderators, and independent, overlapping, and proxy risk factors. Am J Psychiatry 158(6):848-856

15. Prime H, Wade M, Browne DT (2020) Risk and resilience in family wellbeing during the COVID-19 pandemic. Am Psychol 75(5):631-643

16. Rutter M (1979) Protective factors in children's responses to stress and disadvantage. In: Kent MW, Rolf JE (eds) Primary prevention of psychopathology: III. Promoting social competence and coping in children. University Press of New England, Hanover, pp 49-74

17. Gerard JM, Buehler C (2004) Cumulative environmental risk and youth problem behavior. J Marriage Fam 66(3):702-720

18. Wallander J, Berry S, Carr PA, Peterson ER, Waldie KE, Marks E et al (2019) Patterns of exposure to cumulative risk through age 2 and associations with problem behaviors at age 4.5: evidence from growing up in New Zealand. J Abnorm Child Psychol 47(8):1277-1288

19. Crawley E, Loades M, Feder G, Logan S, Redwood S, Macleod J (2020) Wider collateral damage to children in the UK because of the social distancing measures designed to reduce the impact of COVID-19 in adults. BMJ Paediatr Open 4(1):e000701

20. Bonanno GA, Brewin CR, Kaniasty K, Greca AM (2010) Weighing the costs of disaster: consequences, risks, and resilience in individuals, families, and communities. Psychol Sci Public Interest 11(1):1-49

21. Muris P, Merckelbach H, Van Brakel A, Mayer AB (1999) The revised version of the screen for child anxiety related emotional disorders (Scared-R): further evidence for its reliability and validity. Anxiety Stress Coping 12(4):411-425

22. The KIDSCREEN Group Europe (2006) The KIDSCREEN questionnaires - quality of life questionnaires for children and adolescents-handbook. Pabst Science Publishers, Lengerich

23. Spitzer RL, Kroenke K, Williams JBW, Löwe B (2006) A brief measure for assessing generalised anxiety disorder: the GAD-7. Arch Intern Med 166(10):1092-1097

24. Taggart F, Stewart-Brown S, Parkinson J (2015) Warwick-Edinburgh Mental Well-being Scale (WEMWBS) user guide, version 2. NHS Health Scotland, Edinburgh

25. Cohen J, Cohen P, West SG, Aiken LS (2003) Applied multiple regression/correlation analysis for the behavioral sciences, 3rd edn. Lawrence Erlbaum Associates, New York

26. Preacher K (2003) A primer on interaction effects in multiple linear regression. http://www.quantpsy.org/interact/interactions. htm

27. Menard S (1995) Applied logistic regression analysis: Sage University series on quantitative applications in the social sciences. Sage, Thousand Oaks
28. R Core Team [Computer software] (2020) R: a language and environment for statistical computing. R Core Team, Vienna. https:// www.R-project.org/

29. Horikoshi M, Tang Y (2018) ggfortify: data visualisation tools for statistical analysis results. https://CRAN.R-project.org/package= ggfortify

30. Tang Y, Horikoshi M, Li W (2016) ggfortify: unified interface to visualise statistical result of popular $r$ packages. R J 8:474-485

31. Long JA (2020) jtools: analysis and presentation of social scientific data. R Package version 2.1.0. https://cran.r-project.org/packa $\mathrm{ge}=$ jtools

32. Long JA (2019) interactions: comprehensive, user-friendly toolkit for probing interactions. R Package version 1.1.0. https://cran.rproject.org/package $=$ interactions

33. Plummer F, Manea L, Trepel D, McMillan D (2016) Screening for anxiety disorders with the GAD-7 and GAD-2: a systematic review and diagnostic metaanalysis. Gen Hosp Psychiatry 39:24-31

34. Bianca D (2012) Performance of the Warwick-Edinburgh Mental Well-Being Scale (WEMWBS) as a screening tool for depression in UK and Italy. Unpublished Doctoral Dissertation. Università de Bologna, Bologna. http://www2.warwick.ac.uk/fac/med/research/ platform/wemwbs/development/papers/donatella_bianco-thesis.pdf

35. Sprang G, Silman M (2013) Posttraumatic stress disorder in parents and youth after health-related disasters. Disaster Med Public Health Prep 7(1):105-110

36. Moore SA, Faulkner G, Rhodes RE, Brussoni M, Chulak-Brozer T, Ferguson LJ et al (2020) Impact of the COVID-19 virus outbreak on movement and play behaviours of Canadian children and youth: a national survey. Int J Behav Nutr Phys Activity 17:85

37. Pombo A, Luz C, Rodrigues LP, Cordovil R (2021) Effects of COVID-19 confinement on the household routines of children in Portugal. J Child Fam Stud 30:1664-1674

38. Tremblay MS, Gray C, Babcock S, Barnes J, Bradstreet CC, Carr $\mathrm{D}$ et al (2015) Position statement on active outdoor play. Int J Environ Resand Public Health 12:6475-6505

39. Evans GW, Cassells RC (2014) Childhood poverty, cumulative risk exposure, and mental health in emerging adults. Clin Psychol Sci 2(3):287-296

40. Conger RD, Conger KJ, Martin MJ (2010) Socioeconomic status, family processes, and individual development. J Marriage Fam 72(3):685-704

41. Neppl TK, Senia JM, Donnellan MB (2016) Effects of economic hardship: Testing the family stress model over time. J Fam Psychol 30(1):12-21

42. Trentacosta CJ, Hyde LW, Shaw DS, Dishion TJ, Gardner F, Wilson M (2008) The relations among cumulative risk, parenting, and behavior problems during early childhood. J Child Psychol Psychiatry 49:1211-1219

43. Wade M, Prime H, Browne DT (2020) Why we need longitudinal mental health research with children and youth during (and after) the COVID-19 pandemic. Psychiatry Res 290:113143

44. Pereira AI, Muris P, Barros L, Goes R, Marques T, Russo V (2015) Agreement and discrepancy between mother and child in the evaluation of children's anxiety symptoms and anxiety life interference. Eur Child Adolesc Psychiatry 24:327-337

45. Nezlek JB (2008) An introduction to multilevel modeling for social and personality psychology. Soc Personal Psychol Compass 2:842-860

46. Huang F (2018) Multilevel modeling and ordinary least squares regression: how comparable are they? J Exp Educ 86(2):265-281

Publisher's Note Springer Nature remains neutral with regard to jurisdictional claims in published maps and institutional affiliations. 